Endocrinol. Japon. 1963, 10 (1), 35 40

\title{
METHOD FOR THE ASSAY OF THE SUPPRESSIVE ACTION OF CORTICOSTEROIDS ON ADRENAL RESPONSIVENESS
}

\author{
G. FEKETE AND P. GÖRÖG
}

Chemical Works of Gedeon Richter, Ltd., Hormone Department, Budapest, Hungary

Adrenal substitution therapy has for a long time been known to produce adrenal atrophy both in animal experiments and clinical practice (Ingle, 1937; Boland and Headley, 1949). Subsequent studies have thrown light on the mechanism of this action revealing that adrenal atrophy and hypofunction was caused by corticosteroids through the inhibition of ACTH secretion. The question assumed particular significance when cortisone therapy became a feature of everyday practice since with patients given cortisone over prolonged periods, adaptation in stress situations was considerably diminished or ceased altogether. After the synthesis and the introduction of modern corticosteroids (prednisolone, especially dexamethasone) the problem became still more acute. With reference to the latter compound, the first report stated that besides a 190-fold antiphlogistic effect, its inhibitive action on ACTH secretion was 700 times higher than that of hydrocortisone (Arth et al., 1958). This issue is of equal importance from theoretical and practical aspects; from the theoretical point of view : the dissociation of antiphlogistic and pituitary inhibitive effects, in practice: treatment with dexamethasone involves increased danger. This question has since been investigated in the clinical field by numerous authors without any conclusively convincing results; some workers claim that dexamethasone is in fact an extremely potent pituitary inhibitor (Braunsteiner and Schüller, 1959), while others maintain that this side effect runs parallel with the antiphlogistic action (Kelly et al., 1960). With a view to the abovementioned theoretical and practical significance of the matter, it appeared worth while to undertake a thorough inquiry into the question.

In principle, there are several possibilities for the measurement of the inhibitive action of corticosteroids on adrenal function. The latter may be measured indirectly by the aid of ascorbic acid depletion, or directly by measuring steroidogenesis. This process may again be measured in vitro in incubated adrenals, or in vivo in adrenal or peripheral blood or in urine. The factor aliciting increased function may be ACTH or some stressor.

In previous experiments (Fekete, in press; Fekete and Görög, in press) it was found that the ascorbic acid depletion method failed to reflect quantitatively the adrenal reactivity of animals exposed to continued steroid treatment, since the recorded values did not correspond to those obtained by the measurement of steroidogenesis. In the course of these preliminary experiments, experience was

Received for publication October 23, 1962. 
gained in measuring in vitro adrenal steroid production, and this method was found to be easy of execution and readily reproduceable with not too extensive deviations. Therefore, our studies concerned with the development of a routine method for the purpose mentioned above have been based on in vitro corticosterone production under the influence of ACTH.

\section{METHOD}

The experiments in question have been performed on male Wistar rats of our own breed weighing 150 to $200 \mathrm{~g}$. The substance under investigation (hydrocortisone, prednisolone, dexamethasone) were administered once daily over a period of 6 days subcutaneously. Control rats were treated in the same way with the solvent. The various drugs were given in the following doses: hydrocortisone, 1.33 and $4 \mathrm{mg} / 100 \mathrm{~g}$ body weight; prednisolone, 0.2 and $0.6 \mathrm{mg} / 100 \mathrm{~g}$; dexamethasone, 0.04 and $0.12 \mathrm{mg} / 100 \mathrm{~g}$ daily. After treatment of 6 days-on the 7 th daythe animals were anesthesized with $5 \mathrm{mg} / 100 \mathrm{~g}$ intraperitoneal Nembutal and their adrenals removed; the adrenals were cleaned of fatty tissue and treated as follows: In one experiment, the same dose of corticosteroid was given to 2 animals. With each animal, both adrenals were divided into halves and put into 2 jars so that 4 half adrenals were incubated in each jar. All the 3 steroids were studied parallel in each experiment while 4 untreated animals were used as controls, thus the adrenals of 16 animals were involved in every instance.

The adrenals thus divided were incubated for $1 \mathrm{hr}$. in $1.5 \mathrm{ml}$ Krebs-Ringer's solution containing $200 \mathrm{mg} \%$ glucose, at $37^{\circ} \mathrm{C}$, in $95 \% \quad \mathrm{O}_{2}$ and $5 \% \quad \mathrm{CO}_{2}$ atmosphere. After this preincubation, the medium was withdrawn from the tissues, and the adrenals were incubated with a fresh medium containing $27 \mathrm{mU}$ ACTH too, for a period of $2 \mathrm{hrs}$. One $\mathrm{m} l$ of the medium was extracted with methylene-chloride and following evaporation the produced corticosteroids were determined by the aid of blue tetrazolium colour reaction (Elliot et al., 1954). The obtained results have been corrected for the original volume of $1.5 \mathrm{~m} l$ and computed as absolute values (in microgram BT reducing steroids) and expressed in percentile values of the average figures for the controls.

\section{RESULTS}

Table 1. shows the results of the experiments carried out with adrenals of rats treated as described above. From the data contained in Table 1, it is striking

Table 1. Corticosteroid production of adrenals of rats treated with various corticosteroids and of controls

\begin{tabular}{|c|c|c|c|c|c|}
\hline $\begin{array}{l}\text { No. } \\
\text { of } \\
\text { rats }\end{array}$ & Treatment & $\begin{array}{l}\text { Dose } \\
\mathrm{mg} / 100 \mathrm{~g}\end{array}$ & $\begin{array}{c}\text { Adrenal } \\
\text { weight } \\
(\mathrm{mg})^{*}\end{array}$ & $\begin{array}{c}\text { BT reducing } \\
\text { steroids } \\
(\mu \mathrm{g})^{*}\end{array}$ & $\begin{array}{c}\% \\
\text { of } \\
\text { controls }\end{array}$ \\
\hline 12 & - & - & $30.80 \pm 0.67$ & $13.21 \pm 0.88$ & 100.00 \\
\hline 12 & Hydrocortisone & 1.33 & $17.90 \pm 0.44$ & $10.01 \pm 0.46$ & 75.16 \\
\hline 12 & Hydrocortisone & 4.00 & $17.53 \pm 0.38$ & $6.66 \pm 0.53$ & 51.40 \\
\hline 12 & Prednisolone & 0.20 & $26.36 \pm 0.51$ & $11.31 \pm 1.01$ & 85.00 \\
\hline 12 & Prednisolone & 0.60 & $29.58 \pm 0.41$ & $7.72 \pm 0.07$ & 57.85 \\
\hline 12 & Dexamethasone & 0.04 & $19.95 \pm 0.33$ & $8.30 \pm 0.66$ & 62.35 \\
\hline 12 & Dexamethasone & 0.12 & $21.00 \pm 0.46$ & $5.13 \pm 0.40$ & 39.10 \\
\hline
\end{tabular}

$*=$ mean + standard error of the mean (S.E.) 
that inspite of the general reduction in adrenal weights in all the treated groups there is a considerable dissimilarity among the groups treated with various corticosteroids. The greatest decrease in adrenal weights occurs in the hydrocortisone group, the slightest in rats treated with prednisolone, while dexamethasone treated rats lie in-between. The changes in weight thus don't serve quantitative data with respect to the potency of the steroids, as no dose response relationship can be found within the limit of the applied doses. The quantity of the BT reducing corticosteroids on the other hand is closely related to the amount of the steroids administered to the rats. The adrenals of rats treated with higher doses produce a significantly lower quantity of BT reducing material than those treated with lower doses with all the three compounds.

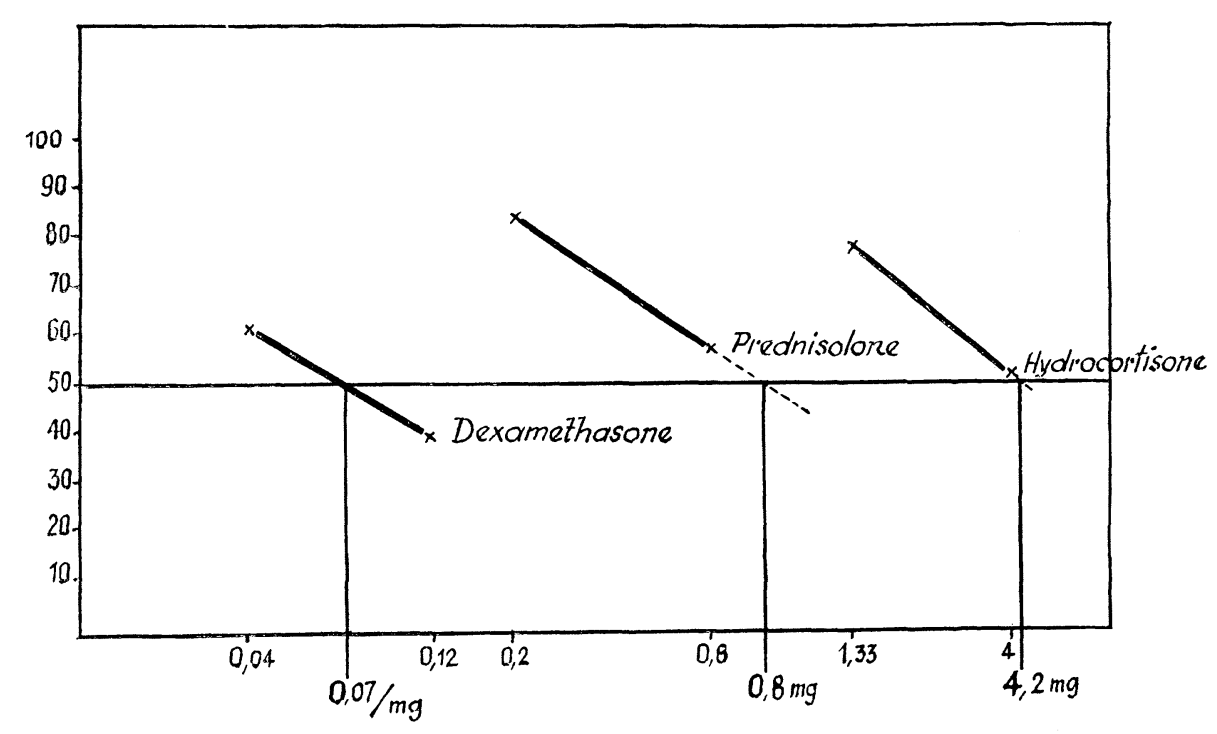

Fig. 1. Dose response curves showing relative adrenal suppressive potencies of hydrocortisone, prednisolone and dexamethasone Ordinate: BT roducing steroids in \% of controls Abscisse : Daily doses of coricosteroids administered in vivo

Applying the above described method for the determination of the corticosteroid amounts bringing about a $50 \%$ reduction of adrenal reactivity in treated animals, it was found that compared to the inhibitive action of hydrocortisone, denoted as 1, that of prednisolone was 4.5 times higher while the corresponding value for dexamethasone was a 59.2-fold one, i.e. the ACTH inhibitive action of dexamethasone was 12.8 times higher than that of prednisolone (Fig. 1). Upon statistical estimation of the results, in evaluating the p-values, calculation was based on the employed relative doses (thus doses of equal antiphlogistic potency) and not on the difference between absolute steroid amounts. 
Evaluation by this method revealed that in relative doses dexamethasone possessed a significantly higher inhibitive activity than either hydrocortisone or prednisolone, whereas, relative doses of prednisolone were on the verge of significance, and were shown to be less active than hydrocortisone. Evaluation of this finding will be resumed in the discussion. $\lambda=\mathrm{S} / \mathrm{b}$ values point to adequate accuracy of the method.

Table 2. Some statistical characteristics of the method described with $p$-values of the difference between the action of equivalent (non/equal) doses of hydrocortisone, prednisolone and dexamethasone

\begin{tabular}{cccrcc}
\hline \hline Compds. & Parallelism & Linearity & \multicolumn{1}{c}{ Rel. potency } & $\lambda=s / b$ & Sign. diff. \\
\hline$D / H$ & $p$ of $\operatorname{dev} \sim 0.30$ & $\mathrm{~F}<1.5$ & $59.2(42.5-93.6)$ & 0.237 & $\mathrm{p}=0.001$ \\
$\mathrm{P} / \mathrm{H}$ & $\mathrm{p}$ of $\operatorname{dev} \sim 0.40$ & $\mathrm{~F}<1.5$ & $4.5(3.1-6.6)$ & 0.227 & $0.01<\mathrm{p}<0.02$ \\
$\mathrm{D} / \mathrm{P}$ & $\mathrm{p}$ of $\operatorname{dev} \sim 0.30$ & $\mathrm{~F}<1.5$ & $12.8(9.3-20.0)$ & 0.208 & $\mathrm{p}<0.001$ \\
\hline
\end{tabular}

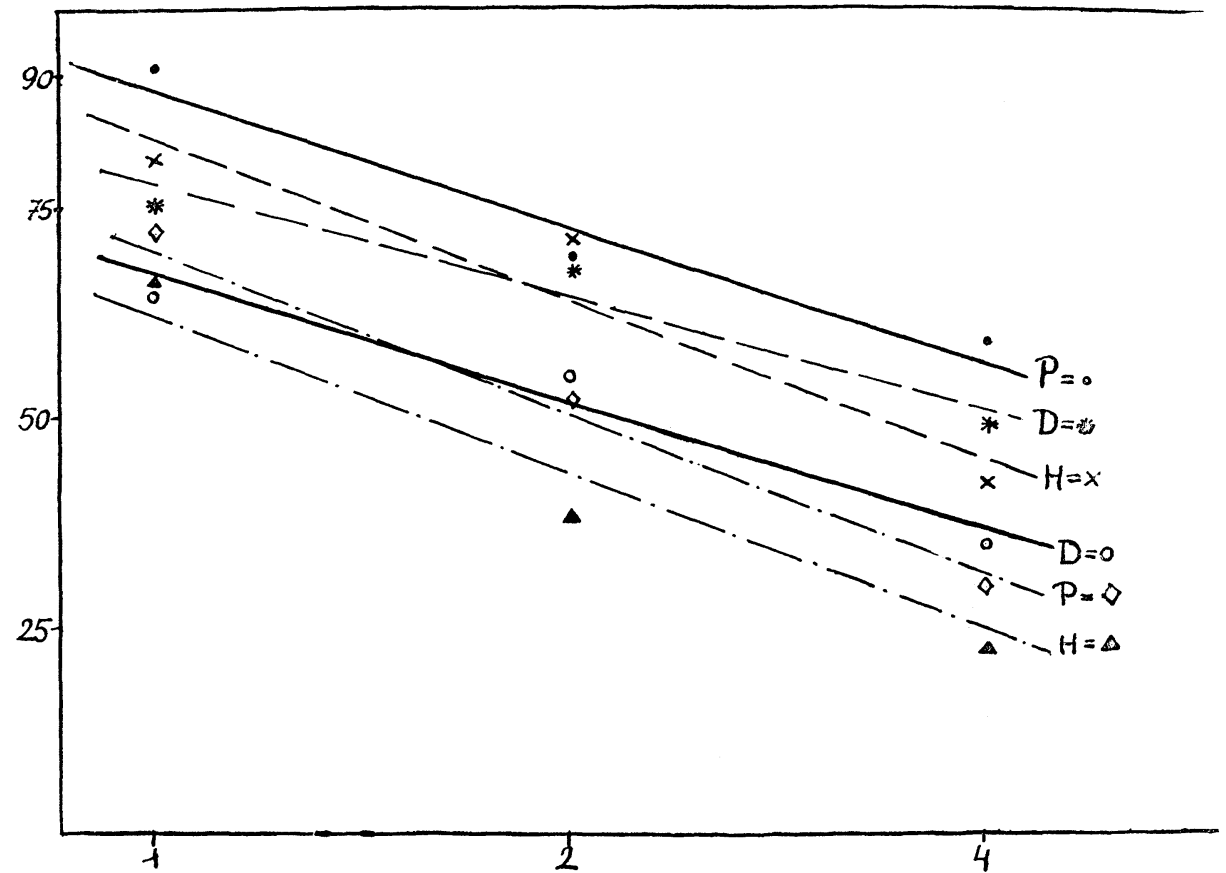

Fig. 2. Regression lines in three six-points-assays showing parallelism and linearity in the dose range applied

The figures shown on the abcissa demonstrate relative doses of equivalent pharmacological potencies.

Ordinate : BT reducing steroids in \% of controls

- comparison of Prednisolon (P) with Dexamethasone (D)

-... comparison of Hydrocortisone $(H)$ with $D$

-..-.- comparison of $\mathrm{H}$ with $\mathrm{P}$ 
Finally the linearity and parallelism of the dose response curves within the limits of the employed doses have also been investigated. For this purpose, three doses of the three steroids in the ratio of $1: 2: 4$ were compared in three separate experiments. In all the three experiments two different steroids were compared according to the possible three variations. The dose response curves are presented in Figure 2. Statistical evaluation of the obtained results (Table 2) demonstrated that the deviation of the dose-effect curves from the parallelism and linearity was improbable as evidenced by $\mathrm{P}$ of deviation and $\mathrm{F}$ values.

\section{DISGUSSION}

As indicated in the introduction, the quantitative investigation of the adrenalsuppressing action due to corticosteroids is equally important from both the theoretical and the practical aspects. Nevertheless, literature includes hardly any report on experiments aimed at the development of such a method. The communication that dexamethasone e.g. has proved to possess a 700 times higher activity than hydrocortisone, is obviously an error due to the inadequacy of the method. The reality of this finding has not been confirmed by clinical experiences, but indirectly Hamburger's (1961) findings also point to irreality. In these experiments intended to avoid hypophysectomy, he had tried the use of prednisolone and dexamethasone; although his results elude accurate quantitative evaluation, they supplied no evidence for confirming such a superior potency in the case of dexamethasone.

Péron and Dorfman (1959) have described a quantitative method for the investigation of the ACTH inhibitive action of corticosteroids. This method is based on determining the adrenal corticosterone content in rats treated with cortisol. Notwithstanding the adequate theoretical basis and the accuracy of their method, we thought it preferable theoretically to develop a method which rested on the study of steroidogenesis and not on checking the steroid content. Our work was furthermore justified by the circumstance that the above cited work demanded the use of such excessive doses of corticosteroids e.g. in the 9- $\alpha$-fluorocortisone group only 1 animal survived treatment.

There can be no doubt that for the investigation of pituitary inhibitive action it is more correct theoretically to activate the pituitary-adrenocortical system by the use of some stressor agent instead of ACTH which admittedly measures reactivity at a lower level (i.e. the adrenals) than is desirable. Moreover, several points argue against this solution.

1. Our own experiments have shown (Fekete, in press) that in the presence of reduced adrenal function, response to stressors cannot be evaluated quantitatively.

2. Owing to the double biological factor (hypophysis-adrenal cortex), the scattering associated with such a method is inevitably wide, if it can be carried out at all.

3. In the last analysis, even if ACTH is used as a stimulant, adrenal reactivity still reflected the measure of previous pituitary inhibition.

With respect to the present results, they clearly demonstrated that under the 
described experimental conditions, dexamethasone has undoubtedly be found to be a more potent pituitary inhibitor than hydrocortisone or prednisolone. This difference is, however, only a double one when compared to pharmacological clinical efficiency.

With the applied relative doses, prednisolone has proved to be less potent than hydrocortisone, which result may be explained by the fact that in our experiments, the prednisolone doses have been somewhat lower than its actual relative efficiency. The obtained 1:4:5 ratio certainly stood nearer to clinical and pharmacological efficiency (Fekete and Szporny, 1961) than the dose ratio of 1:6:6 applied by ourselves.

The adequacy of a pharmacological method in quantitative relations has to be confirmed by practice. Unfortunately, at the present moment, no report is known containing acceptable exact data concerning the relative efficiency of the three steroids as adrenal inhibitors. The available sporadic evidences create the impression that from the clinical point of view our results may be regarded as being actual ones.

\section{SUMMARY}

A new method has been developed for measuring the adreno-cortical suppressive action of corticosteroids. This method rests on measuring in vitro steroidogenesis brought about by a standard dose of ACTH in the adrenals of rats treated with various doses of steroids. According to the obtained results, calculated as pharmacological and therapeutical equivalents, hydrocortisone and prednisolone possess similar efficiency, where, the action of dexamethasone is approximately twice higher.

Accuracy of the method has been checked by the aid of statistical evaluation.

\section{REFERENCES}

Arth, G.E., D.B.R. Johnston, J. Freed, W.W. Spooner, D.R. Hoff and L.H. Sareth (1958). J. Am. Chem. Soc. 80, 3160.

Boland, E.W. and N. Headley (1949). J. Am. Med. Assoc. 141, 301.

Braunsteiner, H. and E. Scüller (1959). Wien. Klin. Wochschr. 71, 598.

Elliott, F.H., M.K. Birmingham, A.V. Schally and E. Schonbaum (1954). Endocrinology 55, 721.

Fekete, G. (1962). Acta Physiol. Acad. Sci. Hung. XXI, 77.

Fekete, G. and P. Görög (1962). Ibid. XXI, 83.

Fekete, G. and L. Szporny (1961). Ibid. XIX, 57.

Hamburger, C. (1960). Acta Endocrinol. 35, 594.

Ingle, D.J. and E.C. Kemdall (1937). Science 86.

Kelly, M.W., C.J. Philip, A.A. Kyriakopoulos, W.J. Bahr and C.E. Bloedow (1960). Arthrit. Rheumat. 3, 129.

Péron, F.G. and R.I. Dorfman (1959). Endocrinology 64, 431. 